\title{
Clinical Outcomes of Biliary Drainage during a Neoadjuvant Therapy for Pancreatic Cancer: Metal versus Plastic Stents
}

\author{
Masaki Kuwatani ${ }^{1}$, Toru Nakamura ${ }^{2}$, Tsuyoshi Hayashi ${ }^{3}$, Yasutoshi Kimura ${ }^{4}$, Michihiro Ono ${ }^{5}$, Masayo Motoya ${ }^{6}$, Koji Imai ${ }^{7}$, \\ Keisuke Yamakita ${ }^{8}$, Takuma Goto ${ }^{9}$, Kuniyuki Takahashi ${ }^{3}$, Hiroyuki Maguchi ${ }^{3}$, Satoshi Hirano ${ }^{2}$, and Hokkaido Pancreatic \\ Cancer Study Group (HOPS) \\ ${ }^{1}$ Department of Gastroenterology and Hepatology, Hokkaido University Hospital, ${ }^{2}$ Department of Gastroenterological Surgery II, Hokkaido \\ University Faculty of Medicine, ${ }^{3}$ Center for Gastroenterology, Teine Keijinkai Hospital, ${ }^{4}$ Department of Surgery, Surgical Oncology and Science \\ and Departments of ${ }^{5}$ Medical Oncology and ${ }^{6}$ Gastroenterology and Hepatology, Sapporo Medical University School of Medicine, Sapporo, \\ ${ }^{7}$ Department of Surgery, and Divisions of ${ }^{8}$ Metabolism and Biosystemic Science and ${ }^{9}$ Gastroenterology and Hematology/Oncology, Asahikawa \\ Medical University, Asahikawa, Japan
}

Neoadjuvant chemotherapy/neoadjuvant chemoradiotherapy (NAC/NACRT) can be performed in patients with pancreatic cancer to improve survival. We aimed to clarify the clinical outcomes of biliary drainage with a metal stent (MS) or a plastic stent (PS) during NAC/NACRT. Between October 2013 and April 2016, 96 patients with pancreatic cancer were registered for NAC/NACRT. Of these, 29 patients who underwent biliary drainage with MS or PS before NAC/NACRT and a subsequent pancreatoduodenectomy were retrospectively analyzed with regard to patient characteristics, preoperative recurrent biliary obstruction rate, NAC/NACRT delay or discontinuation rate, and operative characteristics. The median age of the patients was 67 years. NAC and NACRT were performed in 14 and 15 patients, respectively, and MS and PS were used in 17 and 12 patients, respectively. Recurrent biliary obstruction occurred in $6 \%$ and $83 \%$ of the patients in the MS and PS groups, respectively $(p<0.001)$. NAC/NACRT delay was observed in $35 \%$ and $50 \%$ of the patients in the MS and PS groups, respectively ( $p=0.680)$. NAC/NACRT discontinuation was observed in $12 \%$ and $17 \%$ of the patients in the MS and PS groups, respectively $(p=1.000)$. The operative time in the MS group tended to be longer than that in the PS group (625 minutes vs 497 minutes, $p=0.051$ ), and the operative blood loss volumes and postoperative adverse event rates were not different between the two groups. MS was better than PS from the viewpoint of preventing recurrent biliary obstruction, although MS was similar to PS with regards to perioperative outcomes. (Gut Liver 2020;14:269273)
Key Words: Pancreatic neoplasms; Chemotherapy; Chemoradiotherapy; Jaundice; Stent

\section{INTRODUCTION}

It is controversial whether preoperative biliary drainage is necessary in patients with pancreatic head cancers with jaundice. ${ }^{1,2}$ Some reports have revealed that preoperative biliary drainage when waiting for curative surgery can increase biliary adverse events, whereas others have indicated the necessity of biliary drainage in the case of severe jaundice and neoadjuvant chemotherapy/neoadjuvant chemoradiotherapy (NAC/NACRT). NAC/NACRT for resectable or borderline resectable pancreatic cancers has recently been initiated to improve clinical outcomes in some institutions because curative resection followed by adjuvant chemotherapy cannot sufficiently improve the survival (5-year survival rate of $<6 \%$ in 2015). ${ }^{5}$ Some clinical studies have reported that NAC/NACRT is beneficial compared with upfront surgery for pancreatic cancer as well as other gastrointestinal cancers. ${ }^{6}$

There have been many reports on biliary drainage for patients with unresectable pancreatic cancer, which have indicated that a metal stent (MS) is superior to a plastic stent (PS) in terms of patency; ${ }^{7}$ however, there have been few reports on biliary drainage for patients with pancreatic cancer during NAC/NACRT. A meta-analysis on preoperative biliary drainage with/without NAC/NACRT showed that the rates of endoscopic re-intervention before surgery and the occurrence rates of postoperative

Correspondence to: Masaki Kuwatani

Department of Gastroenterology and Hepatology, Hokkaido University Hospital, North 14, West 5, Kita-ku, Sapporo 060-8648, Japan

Tel: +81-11-716-1161 (ext. 5918), Fax: +81-11-706-7867, E-mail: mkuwatan@med.hokudai.ac.jp

Received on December 24, 2018. Revised on March 10, 2019. Accepted on March 24, 2019. Published online November 4, 2019. pISSN 1976-2283 eISSN 2005-1212 https://doi.org/10.5009/gnl18573

(c) This is an Open Access article distributed under the terms of the Creative Commons Attribution Non-Commercial License (http://creativecommons.org/licenses/by-nc/4.0) which permits unrestricted non-commercial use, distribution, and reproduction in any medium, provided the original work is properly cited. 
complications, especially pancreatic fistula, were higher in the PS group than in the MS group. ${ }^{8}$ For biliary decompression during NAC/NACRT, we also need to consider removability of a biliary stent (easier to remove PS than MS) and total stenting cost (cheaper single PS than single MS).

We also performed two multicenter prospective observational studies with NAC/NACRT: NAC with two cycles of S-1 for patients with resectable pancreatic cancer; NACRT with radiation and S-1 followed by three cycles of gemcitabine in cooperation with radiologists for patients with borderline resectable (locally advanced) pancreatic cancer. In these studies, some patients underwent biliary decompression with MS or PS for NAC/NACRT followed by pancreatoduodenectomy. Thus, we retrospectively performed a subanalysis of this population to clarify the clinical outcomes of biliary drainage with MS and PS during NAC/ NACRT.

\section{CASE REPORT}

From October 2013 to April 2016, 96 patients with pathologically diagnosed pancreatic cancer who gave informed consent were registered for NAC or NACRT studies conducted by Hokkaido Pancreatic Cancer Study Group (HOPS) (UMIN000013031/ UMIN000012293). Of these, 29 patients who underwent biliary drainage with MS or PS before NAC/NACRT and subsequent pancreatoduodenectomy were analyzed.

Patients with resectable pancreatic cancer were registered in HOPS-R01, in which NAC with S-1 (80 mg/m², twice daily) for two cycles (84 days) was performed. After NAC, patients underwent curative surgery within 6 weeks after last oral administration of S-1. Patients with borderline resectable pancreatic cancer based on National Comprehensive Cancer Network guidelines (2012, version 2) were registered in HOPS-BR01, in which NACRT with a total dose of 50.4 Gy in 28 fractions and S-1 (80 $\mathrm{mg} / \mathrm{m}^{2}$, twice daily on radiation day alone) was continued for 1 month with subsequent chemotherapy with three cycles of gemcitabine (84 days). After NACRT, patients underwent curative surgery within 8 weeks after last administration of gemcitabine. Before NAC/NACRT, patients with total bilirubin $>2.0 \mathrm{mg} / \mathrm{dL}$ or $>3$ times the aspartate aminotransferase/alanine aminotransferase value of the normal upper limit underwent endoscopic biliary stenting with MS or PS at the discretion of an endoscopist.

Recurrent biliary obstruction (RBO) after endoscopic biliary stenting was defined on the basis of the Tokyo criteria. ${ }^{9}$ Adverse events related to endoscopic biliary stenting were defined on the basis of a lexicon of American Society for Gastrointestinal Endoscopy. ${ }^{10}$ When NAC/NACRT could not be performed on the planned date due to adverse events and performed on another date, it was defined as NAC/NACRT delay. When NAC/NACRT was canceled due to adverse events or patient refusal, it was defined as NAC/NACRT discontinuation. Complications after curative surgery were evaluated and graded on the basis of the extended Clavien-Dindo classification. ${ }^{11}$ Postoperative pancreatic fistula was defined according to the International Study Group definition. ${ }^{12}$

Unresectable conversion rate in NAC was eight out of 51 $(16 \%)(8 / 49$ [16\%] after exclusion of ineligible 2 cases) and that in NACRT, 17 out of 45 (38\%). Patient characteristics of the 29 patients are shown in Table 1 . Twenty-two patients were classified as Union for International Cancer Control stage IIA and seven patients, as stage III. Further, 14 patients underwent NAC, and 15 patients underwent NACRT. For biliary decompression, MS and PS were placed in 17 and 12 patients, respectively. The details of MS/PS stent are indicated in Table 1.

RBO occurred in 11 patients, of which one patient was in the MS group and 10 patients including two migration cases were in the PS group. The occurrence rate of RBO was significantly higher in the PS group (83.3\%) than in the MS group (6\%) $(\mathrm{p}<0.001)$ as well as the average stenting number per patient (MS/PS, 1.12/2.08) $(\mathrm{p}<0.001)$. The log-rank test also revealed that the patency in the MS group was significantly higher than that in the PS group (not reached vs 63.5 days, p<0.001) (Fig. 1). There was no stenting-related adverse event which affected NAC/NACRT.

NAC/NACRT delay was observed in 35\% of the patients in the MS group and 50\% of the patients in the PS group; NAC/ NACRT discontinuation, in $12 \%$ and 17\%, respectively. Mean relative dose intensities of NAC and NACRT were respectively 87\% and 98\% (the first part with S-1 with radiotherapy) followed by $74 \%$ (the second part with gemcitabine) in total: $95 \%$ and $98 \%$ followed by $68 \%$ in the MS group, while 76\% and $100 \%$ followed by $83 \%$ in the PS group.

The operative time in the MS group tended to be longer than that in the PS group (625 minutes vs 497 minutes, $\mathrm{p}=0.051$ ), while operative blood loss, postoperative adverse event rates including postoperative pancreatic fistula and the distributions of Evans classification in both groups were not significantly different.

A multiple regression analysis for operative times was performed. Of the 10 factors (age, sex, final tumor size, Union for International Cancer Control stage, stent type, stent patency, RBO, neoadjuvant therapy, operative blood loss, and Evans classification), sex, stent type, neoadjuvant therapy, and operative blood loss significantly contributed to operative time $\left(R^{2}=0.69\right)$ (regression coefficient \pm standard error, p-value: $0.061 \pm 0.0264$ $\mathrm{p}=0.029 ;-0.0639 \pm 0.0261, \mathrm{p}=0.023 ;-0.0985 \pm 0.0253, \mathrm{p}<0.001$ $0.0000902 \pm 0.0000241, \mathrm{p}=0.001)$.

\section{DISCUSSION}

The present study revealed that MS is better than PS for biliary decompression during NAC/NACRT from the viewpoint of preventing RBO.

Many studies have revealed the superiority of MSs to PSs for 
Table 1. Characteristics of the 29 Patients

\begin{tabular}{|c|c|c|c|c|}
\hline Characteristic & All & MS & PS & p-value (MS vs PS) \\
\hline No. of patients & 29 & 17 & 12 & - \\
\hline Male/female & $13 / 16$ & $8 / 9$ & $5 / 7$ & 1.000 \\
\hline Age, median (range), yr & $67(50-83)$ & $66(50-83)$ & $68(53-80)$ & 0.824 \\
\hline JPS stage (6th) I/II/III/IV & 0/0/10/19 & $0 / 0 / 5 / 12$ & $0 / 0 / 5 / 7$ & 1.000 \\
\hline UICC stage (7th) IIA/III & $22 / 7$ & $12 / 5$ & $10 / 2$ & 0.665 \\
\hline NAC/NACRT, mean RDI (\%) & $14 / 15\left(87 / 98^{*}, 74^{\dagger}\right)$ & $8 / 9\left(95 / 98^{*}, 68^{\dagger}\right)$ & $6 / 6\left(76 / 100^{*}, 83^{\dagger}\right)$ & - \\
\hline Delay, \% & & 35 & 50 & 0.680 \\
\hline Discontinuation, $\%$ & & 12 & 17 & 1.000 \\
\hline Final tumor size, median (range), $\mathrm{mm}^{\ddagger}$ & $20(11-40)$ & $21(14-40)$ & $19(11-28)$ & 0.351 \\
\hline RBO rate, \% (migration no.) & $38(2)$ & $6(0)$ & $83(2)$ & $<0.001$ \\
\hline Stenting cost per patient (US \$) & & $4,973^{\S}$ & $5,700^{\S}$ & - \\
\hline Stent diameter/no. of patients & & $10 \mathrm{~mm} / 17$ & $7 \mathrm{~F} / 9,8.5 \mathrm{~F} / 2,10 \mathrm{~F} / 1$ & - \\
\hline Stent length 5/6/7/8 cm & & $1 / 13 / 0 / 3$ & $4 / 0 / 8 / 0$ & - \\
\hline Fully-/partially-/uncovered & & $5 / 12 / 0$ & - & - \\
\hline ES operation +/- & $12 / 17$ & $5 / 12$ & $7 / 5$ & 0.148 \\
\hline Stenting-related AE & 1 & $1^{\prime \prime}$ & 0 & 1.000 \\
\hline Operative procedure SSPPD/TP & & $17 / 0$ & $11 / 1$ & 1.000 \\
\hline Operative time, median (IQR), min & & $625(493-694)$ & $497(397-554)$ & 0.051 \\
\hline Operative blood loss, median (IQR), mL & & $719(430-1,150)$ & $649(301-927)$ & 0.479 \\
\hline Postoperative AE, \% & & 47.1 & 50 & 1.000 \\
\hline Clavian-Dindo classification I/II/IIIa & & $3 / 3 / 2$ & $0 / 2 / 4$ & 0.353 \\
\hline Evans classification $\mathrm{I} / \mathrm{II} / \mathrm{IIb} / \mathrm{III}$ & & $4 / 4 / 8 / 1$ & $4 / 6 / 2 / 0$ & 0.243 \\
\hline
\end{tabular}

MS, metal stent; PS, plastic stent; JPS, Japan Pancreas Society; UICC, Union for International Cancer Control; NAC, neoadjuvant chemotherapy; NACRT, neoadjuvant chemoradiotherapy; RDI, relative dose intensity; RBO, recurrent biliary obstruction; ES, endoscopic sphincterotomy; AE, adverse event; SSPPD, subtotal stomach-preserving pancreaticoduodenectomy; TP, total pancreatectomy; IQR, interquartile range.

${ }^{*}$ First part with S-1 with radiotherapy; ${ }^{\dagger}$ Second part with gemcitabine; ${ }^{\ddagger}$ The size measured on contrast-enhanced computed tomography within 2

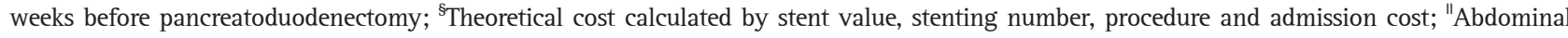
pain after stenting. Categorical data were examined using the chi-square test. The Mann-Whitney U test was used to compare quantitative data.

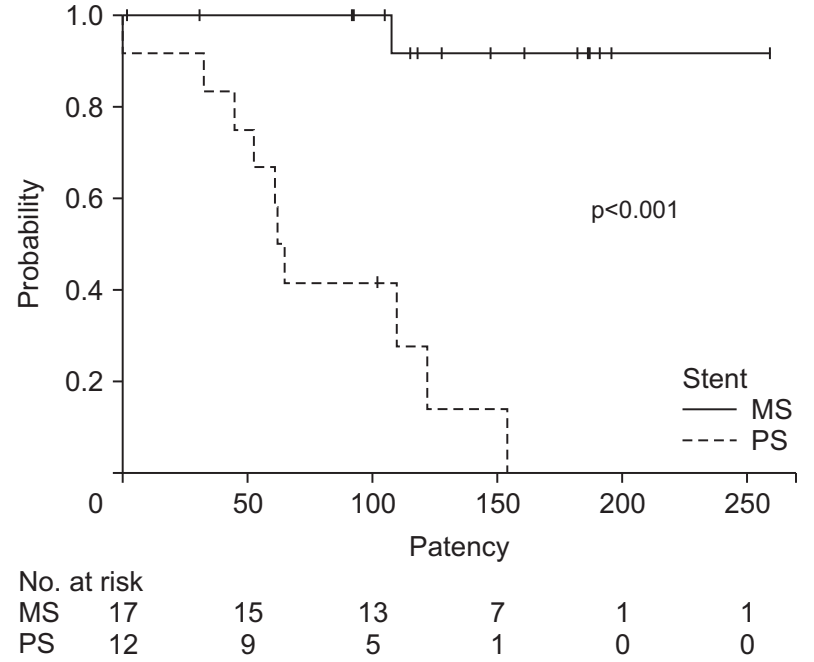

Fig. 1. Kaplan-Meier curves for stent patency according to the stents used. The patency in the metal stent (MS) group was significantly higher than that in the plastic stent (PS) group. stent patency in patients with both unresectable and resectable malignant distal biliary strictures, and this was compatible with our result. ${ }^{4,13-15}$ This superiority may result from the larger diameter of MSs than that of PSs.

NAC/NACRT is becoming increasingly important for the improvement of clinical outcomes after the resection of pancreatic cancers. Some previous studies have indicated a better prognosis for pancreatic cancer patients who undergo NAC/NACRT ${ }^{16,17}$ than for those who do not, and some randomized controlled trials comparing NAC with NACRT and comparing NAC/NACRT with up-front surgery for pancreatic cancers are ongoing. ${ }^{18-20}$ The roles of biliary decompression, drainage method, and choice of the biliary stent during NAC/NACRT are very significant during the preoperative period. The retrospective study by Tsuboi et al. ${ }^{21}$ revealed that MS implantation is effective for preoperative biliary drainage in pancreatic cancer cases treated with NAC because of the low rates of stent-related complications ( $0 \%$ vs 
72.7\%) and NAC delay (11.1\% vs $81.8 \%$ ).

How MS or PS pathologically affects the bile duct, vessels, duodenal mucosa, and the tissues around them during NAC/NACRT is unclear. According to some reports including operative and autopsy cases, remarkable collagenous reaction, inflammation, and epithelial atypia in the bile duct occur around uncovered MSs in patients with malignant biliary obstruction. ${ }^{22,23}$ Such pathological changes would occur in the case of NAC/NACRT with MSs and negatively affect the operative time and hemorrhagic volume during surgery. There have been some reports concerning the relationship between radiation and pathological response of organs, indicating that sclerosis and stricture can occur in the bile duct, whereas ulcer, bleeding, and perforation by ischemia can occur in the gastrointestinal tract. ${ }^{24,25}$ Thus, MS and radiation could synergistically cause pathological damage to the bile duct and duodenum in the radiation area, leading to perioperative bleeding and prolonged operative time. Meanwhile, some previous reports have indicated that preoperative placement of a MS does not affect clinical outcomes concerning the resection of pancreatic cancers, including adverse events during the perioperative period, and that MS is superior to PS with regard to preoperative stent patency. ${ }^{4,15}$ Thus, MS would be appropriate for preoperative biliary decompression during NAC/ NACRT, although there has been no randomized controlled trial comparing MS with PS during NAC/NACRT.

There are some limitations to the present study. First, this was a retrospective, non-randomized study with an insufficient number of patients. Second, neoadjuvant therapy included both chemotherapy and chemoradiotherapy. Third, the size of PS was not unified.

In conclusion, MS would be better than PS from the viewpoint of preventing RBO, although MS would be similar to PS with regard to perioperative outcomes.

\section{CONFLICTS OF INTEREST}

No potential conflict of interest relevant to this article was reported.

\section{ACKNOWLEDGEMENTS}

We deeply appreciate the support of Dr. Yusuke Sakuhara, Dr. Hiroshi Kawakami, Dr. Kazumichi Kawakubo, Dr. Kimitoshi Kubo, Dr. Shuhei Kawahata, Dr. Shin Kato, Dr. Itsuki Sano, Dr. Ryo Sugiura, Dr. Naoya Sakamoto, Dr. Keisuke Okamura, Dr. Takahiro Tsuchikawa, Dr. Eiji Tamoto, Dr. Takehiro Noji, Dr. Toshimichi Asano, Dr. Yoshitsugu Nakanishi, Dr. Hiroki Saito, Dr. Kimitaka Tanaka, and Dr. Takashi Ueno from Hokkaido University Hospital; Dr. Makoto Yoshida, Dr. Michihiro Ono, Dr. Masahiro Shitani, Dr. Masafumi Imamura, and Dr. Minoru Nagayama from Sapporo Medical University; Dr. Takahide Sasaki, Dr. Tomohide Nakayama, and Dr. Eiichi Tanaka from
Hokkaido Gastroenterology Hospital; Dr. Akio Katanuma, Dr. Kei Yane, Dr. Toshifumi Kin, Dr. Yoshiyasu Anbo, Dr. Minoru Takada, and Dr. Kentaro Kato from Teine Keijinkai Hospital; Dr. Jo Matsumoto from Obihiro Kosei Hospital; Dr. Hiroyuki Kaneto and Dr. Kenichi Sasaki from Muroran City General Hospital.

\section{AUTHOR CONTRIBUTIONS}

Study design: M.K. Data acquisition: M.K., T.N., T.H., Y.K., M.O., M.M., K.I., K.Y., T.G., K.T. Data analysis: M.K. Drafting of the manuscript: M.K. Critical revision of the manuscript: M.K., T.N., T.H., Y.K., M.O., M.M., K.I., K.Y., T.G., K.T. Study supervision: H.M., S.H. Approval of final manuscript: all authors.

\section{ORCID}

Masaki Kuwatani

Toru Nakamura

Tsuyoshi Hayashi

Yasutoshi Kimura

Michihiro Ono

Masayo Motoya

Koji Imai

Keisuke Yamakita

Takuma Goto

Kuniyuki Takahashi

Hiroyuki Maguchi

Satoshi Hirano https://orcid.org/0000-0002-0265-6068 https://orcid.org/0000-0003-3999-9627 https://orcid.org/0000-0001-7666-5795 https://orcid.org/0000-0002-7790-9250 https://orcid.org/0000-0001-9015-0069 https://orcid.org/0000-0003-2566-0382 https://orcid.org/0000-0001-9318-1450 https://orcid.org/0000-0001-7278-712X https://orcid.org/0000-0002-7198-3038 https://orcid.org/0000-0002-4989-0863 https://orcid.org/0000-0001-6807-5613 https://orcid.org/0000-0003-2612-9913

\section{REFERENCES}

1. Scheufele F, Schorn S, Demir IE, et al. Preoperative biliary stenting versus operation first in jaundiced patients due to malignant lesions in the pancreatic head: a meta-analysis of current literature Surgery 2017;161:939-950.

2. Moole H, Bechtold M, Puli SR. Efficacy of preoperative biliary drainage in malignant obstructive jaundice: a meta-analysis and systematic review. World J Surg Oncol 2016;14:182.

3. Lutz MP, Zalcberg JR, Ducreux M, et al. 3rd St. Gallen EORTC Gastrointestinal Cancer Conference: consensus recommendations on controversial issues in the primary treatment of pancreatic cancer. Eur J Cancer 2017;79:41-49.

4. Tol JA, van Hooft JE, Timmer R, et al. Metal or plastic stents for preoperative biliary drainage in resectable pancreatic cancer. Gut 2016;65:1981-1987.

5. Siegel RL, Miller KD, Jemal A. Cancer statistics, 2015. CA Cancer J Clin 2015;65:5-29.

6. Heinrich S, Lang H. Neoadjuvant therapy of pancreatic cancer: definitions and benefits. Int J Mol Sci 2017;18:E1622.

7. Almadi MA, Barkun A, Martel M. Plastic vs. self-expandable metal stents for palliation in malignant biliary obstruction: a series of meta-analyses. Am J Gastroenterol 2017;112:260-273. 
8. Crippa S, Cirocchi R, Partelli S, et al. Systematic review and metaanalysis of metal versus plastic stents for preoperative biliary drainage in resectable periampullary or pancreatic head tumors. Eur J Surg Oncol 2016;42:1278-1285.

9. Isayama H, Hamada T, Yasuda I, et al. TOKYO criteria 2014 for transpapillary biliary stenting. Dig Endosc 2015;27:259-264.

10. Cotton PB, Eisen GM, Aabakken L, et al. A lexicon for endoscopic adverse events: report of an ASGE workshop. Gastrointest Endosc 2010;71:446-454.

11. Katayama H, Kurokawa Y, Nakamura K, et al. Extended ClavienDindo classification of surgical complications: Japan Clinical Oncology Group postoperative complications criteria. Surg Today 2016;46:668-685.

12. Bassi C, Marchegiani G, Dervenis C, et al. The 2016 update of the International Study Group (ISGPS) definition and grading of postoperative pancreatic fistula: 11 years after. Surgery 2017;161:584591.

13. Moss AC, Morris E, Mac Mathuna P. Palliative biliary stents for obstructing pancreatic carcinoma. Cochrane Database Syst Rev 2006;(2):CD004200.

14. Sawas T, Al Halabi S, Parsi MA, Vargo JJ. Self-expandable metal stents versus plastic stents for malignant biliary obstruction: a meta-analysis. Gastrointest Endosc 2015;82:256-267.

15. Olsson G, Frozanpor F, Lundell L, et al. Preoperative biliary drainage by plastic or self-expandable metal stents in patients with periampullary tumors: results of a randomized clinical study. Endosc Int Open 2017;5:E798-E808.

16. Rose JB, Rocha FG, Alseidi A, et al. Extended neoadjuvant chemotherapy for borderline resectable pancreatic cancer demonstrates promising postoperative outcomes and survival. Ann Surg Oncol 2014;21:1530-1537.

17. Torgeson A, Garrido-Laguna I, Tao R, Cannon GM, Scaife CL, Lloyd S. Value of surgical resection and timing of therapy in patients with pancreatic cancer at high risk for positive margins. ESMO Open 2018;3:e000282.

18. Katz MHG, Ou FS, Herman JM, et al. Alliance for clinical trials in oncology (ALLIANCE) trial A021501: preoperative extended chemotherapy vs. chemotherapy plus hypofractionated radiation therapy for borderline resectable adenocarcinoma of the head of the pancreas. BMC Cancer 2017;17:505.

19. Tachezy M, Gebauer F, Petersen C, et al. Sequential neoadjuvant chemoradiotherapy (CRT) followed by curative surgery vs. primary surgery alone for resectable, non-metastasized pancreatic adenocarcinoma: NEOPA. A randomized multicenter phase III study (NCT01900327, DRKS00003893, ISRCTN82191749). BMC Cancer 2014;14:411.

20. Versteijne E, van Eijck CH, Punt CJ, et al. Preoperative radiochemotherapy versus immediate surgery for resectable and borderline resectable pancreatic cancer (PREOPANC trial): study protocol for a multicentre randomized controlled trial. Trials 2016;17:127.

21. Tsuboi T, Sasaki T, Serikawa M, et al. Preoperative biliary drainage in cases of borderline resectable pancreatic cancer treated with neoadjuvant chemotherapy and surgery. Gastroenterol Res Pract 2016;2016:7968201.

22. Bethge N, Sommer A, Gross U, von Kleist D, Vakil N. Human tissue responses to metal stents implanted in vivo for the palliation of malignant stenoses. Gastrointest Endosc 1996;43:596-602.

23. Euscher ED, Marsh WL Jr, Lucas JG, Frankel WL. Histologic and immunohistochemical changes in the stented common bile duct. Appl Immunohistochem Mol Morphol 2007;15:299-304.

24. Pollom EL, Chin AL, Diehn M, Loo BW, Chang DT. Normal tissue constraints for abdominal and thoracic stereotactic body radiotherapy. Semin Radiat Oncol 2017;27:197-208.

25. Maturen KE, Feng MU, Wasnik AP, et al. Imaging effects of radiation therapy in the abdomen and pelvis: evaluating "innocent bystander" tissues. Radiographics 2013;33:599-619. 